\title{
Multifunctional oxides for integrated manufacturing of efficient graphene electrodes for organic electronics
}

\author{
Piran R. Kidambi, ${ }^{1 \ddagger}$ Christ Weijtens, ${ }^{2}$ John Robertson, ${ }^{1}$ Stephan Hofmann ${ }^{1}$ and Jens Meyer ${ }^{2 a)}$ \\ ${ }^{1}$ Department of Engineering, University of Cambridge, 9 JJ Thomson Avenue, Cambridge CB3 OFA, UK \\ ${ }^{2}$ Philips Research, Philipsstrasse 8, 52068 Aachen, Germany \\ "Current address: Department of Mechanical Engineering, Massachusetts Institute of Technology, 77 \\ Massachusetts Avenue, Cambridge, MA 02139-4307 \\ \#electronic mail: jens.meyer@philips.com
}

\begin{abstract}
Using multi-functional oxide films, we report on the development of an integration strategy for scalable manufacturing of graphene-based transparent conducting electrodes (TCE) for organic electronics. A number of fundamental and process challenges exist for efficient graphene-based TCEs, in particular environmentally and thermally stable doping, interfacial band engineering for efficient charge injection/extraction, effective wetting and process compatibility including masking and patterning. Here, we show that all of these challenges can be effectively addressed at once via a thin (>10 nm) metal oxide $\left(\mathrm{MoO}_{3}\right.$ or $\left.\mathrm{WO}_{3}\right)$ coating of the graphene. We demonstrate graphene electrode patterning without the need for conventional lithography and thereby achieve organic light emitting diodes (OLEDs) with efficiencies exceeding those of standard ITO reference devices.
\end{abstract}

Keywords: graphene, $\mathrm{WO}_{3}, \mathrm{MoO}_{3}$, doping, patterning, OLED 
Scalable growth, transfer and novel front/back-end integration strategies are imperative for the development of successful commercialization avenues for graphene based technologies. ${ }^{1,2}$ The use of graphene as a potential replacement of brittle and increasingly expensive indium tin oxide (ITO) as a transparent conducting electrode (TCE) in organic electronics and optoelectronic applications has attracted a lot of research interest, ${ }^{3-6}$ but effective integration into working devices remains challenging. ${ }^{1,2}$ While graphene's high optical transparency ( 2.3\% absorption in the visible range) and charge carrier mobility $>15000 \mathrm{~cm}^{2} \mathrm{~V}^{-1} \mathrm{~s}^{-1}$ are intrinsic material advantages, ${ }^{7}$ its high flexibility (strain of up to $20 \%$ ) can be exploited as a key functional competitive advantage over ITO for future bendable or elastic optoelectronic applications. ${ }^{2}$ Organic light emitting diodes (OLEDs) are among several key applications that would benefit significantly from such graphene based TCEs. $^{2,8-11}$ While chemical vapor deposition (CVD) has emerged as the preferred technique for large area graphene synthesis $^{12-15}$ and roll-to-roll integrated graphene transfer over large areas has been demonstrated, ${ }^{16,17}$ a number of fundamental and process challenges remain for efficient graphene-based TCEs: (1) environmentally and thermally stable doping, ${ }^{18,19}$ (2) interfacial band engineering for efficient charge injection/extraction, ${ }^{18,19}$ (3) effective wetting, ${ }^{9,20,28}$ and (4) process compatibility including masking and patterning. Recently, we have shown that metal oxides $\left(\mathrm{WO}_{3}, \mathrm{~V}_{2} \mathrm{O}_{5}\right.$ and $\left.\mathrm{MoO}_{3}\right)$ can effectively p-dope graphene. ${ }^{9,18,19}$ The doping is not only environmentally stable, but also withstands elevated temperatures beyond $100^{\circ} \mathrm{C}$ which is a clear advantage compared to conventional doping approaches, e.g. nitride acids. ${ }^{17,21,22}$ In addition, the metal oxides allow for an efficient charge injection from graphene into the OLED due to a favorable energy matching of the transport levels. ${ }^{9,18,19}$ However, industrial applications also require integrated process solutions [(3) and (4)]. Lithography is commonly used to pattern graphene, which however is relatively expensive and cannot be directly integrated in large scale roll-to-roll manufacturing processes. ${ }^{23-26}$ Further, lithography typically involves the use of solvents and chemicals that might affect or degrade the properties of doped graphene..$^{8-11,21,27-31}$

Here, we show that all of these challenges for efficient graphene-based TCEs can be effectively addressed at once via a thin $(>10 \mathrm{~nm})$ metal oxide $\left(\mathrm{MoO}_{3}\right.$ or $\left.\mathrm{WO}_{3}\right)$ coating of the graphene. The metal oxide can be deposited by various means, ${ }^{9,18,19}$ but we focus here on thermal evaporation. The oxide coating provides effective graphene doping, ideal alignment of the transport levels at the graphene interface, effective wetting and graphene protection during oxygen plasma etching and patterning. Raman analysis confirms the protective nature of these thin $(>10 \mathrm{~nm})$ metal oxide $\left(\mathrm{MoO}_{3}\right.$ or $\left.\mathrm{WO}_{3}\right)$ coatings on graphene. This multi-functionality opens up effective integrated graphene manufacturing routes. We demonstrate graphene electrode patterning without the need for conventional lithography and thereby achieve OLEDs with efficiencies exceeding those of standard ITO reference devices. 
Figure 1(a) schematically highlights the concept of the entire process. Graphene synthesis was performed on commercially available $\mathrm{Cu}$ foils ${ }^{12,13,15}$ using a low pressure chemical vapor deposition process ${ }^{32}$ and subsequently transferred to glass or $\mathrm{SiO}_{2}(300 \mathrm{~nm}) / \mathrm{Si}$ wafer substrates using PMMA as a carrier layer. ${ }^{24}$ Post transfer the samples were annealed in 4 mbar $\mathrm{H}_{2}$ at $\sim 300-350^{\circ} \mathrm{C}$ for $60 \mathrm{~min}$ to remove polymer residue. ${ }^{18,19}$ This is followed by thermal deposition of $\mathrm{WO}_{3}$ through a shadowmask which induces p-doping of graphene due to charge transfer. ${ }^{18,19}$ An $\mathrm{O}_{2}$ plasma step is then used to etch the regions that are not covered by $\mathrm{WO}_{3}$, while the covered graphene areas are protected against etching leading to the desired pattern formation (Figure 1(b)). An OLED stack with a perpendicular patterned top electrode is evaporated on top of the $\mathrm{WO}_{3}$ covered graphene stripes. The intersections of the patterned graphene and top electrode defines the active area of the OLED. Figure 1(c) shows the corresponding operating OLED with a clearly defined active area. Note that the shadow mask technology allows for processing of high resolution OLED displays (< 5 um pixel size). Our process is generally applicable to mono- and few-layer material, but we focus here on monolayer graphene, which is most sensitive to potential damage and other deleterious effects.

We first use electrical measurements (Four-Point-Probe with a probe distance of $1 \mathrm{~mm}$ ) to quantify the damage to graphene during an $\mathrm{O}_{2}$ plasma treatment at 100W. Figure 2(a) shows the change in sheet resistance of monolayer graphene transferred to a $\mathrm{Si} / \mathrm{SiO}_{2}(300 \mathrm{~nm})$ wafer as a function of the $\mathrm{O}_{2}$ plasma exposure time. While a short exposure of $\sim 10 \mathrm{~s}$ already leads to an order of magnitude increase in sheet resistance, a 60s plasma completely destroys the graphene layer. Figures 2(b) and 3 show what happens when the graphene is covered by a thin oxide film. The metal oxide deposition leads to an abrupt and substantial lowering of sheet resistance of the graphene layer (Figure 2(b)). This can be explained by a charge transfer process of electrons from graphene to $\mathrm{WO}_{3}$. Recently, we have shown by photoemission spectroscopy studies that a large dipole exists at the interface between graphene and the metal oxide as a result of a large work function (WF) difference. ${ }^{18,19}$ When both materials are brought in contact Fermi level alignment across the interface takes place compensating the very the WF difference of graphene $(\mathrm{WF} \approx 4.4 \mathrm{eV})$ and $\mathrm{WO}_{3}(\mathrm{WF} \approx 6.7 \mathrm{eV})^{31}$, as schematically illustrated in the inset of Fig $2(\mathrm{~b})$. This charge transfer process induces p-doping of graphene as a result of Fermi level alignment across the interface. Therefore the sheet resistance decreases rapidly with increasing oxide thickness to a value of $<300 \Omega / \mathrm{sq}$, until a closed oxide coverage of $30-50 \AA$ is formed (see also AFM data in Figure 3(c) and prior TEM images reported elsewhere). ${ }^{18,19}$ We note that our graphene samples are already p-doped due to ambient air-exposure and PMMA residues prior to the $\mathrm{WO}_{3}$ deposition. For comparison, an intrinsic monolayer has a sheet resistance in the order of $6 \mathrm{k} \Omega /$ sq. Figure 3 highlights the protective effect of this oxide coating during $\mathrm{O}_{2}$ plasma exposure. We use Raman spectroscopy to characterize the change in quality of the oxide covered graphene by monitoring the change in the ratio of the intensity of the D peak $\sim 1350 \mathrm{~cm}^{-1}$ and the $\mathrm{G}$ peak $\sim 1600 \mathrm{~cm}^{-1}$ 
$\left(\mathrm{I}_{\mathrm{D}} / \mathrm{I}_{\mathrm{G}}\right.$ ratio). ${ }^{12}$ In addition to damage we observed that graphene is removed upon $\mathrm{O}_{2}$-plasma exposure most likely due to oxidation from the $\mathrm{O}_{2}$-plasma. The changes in the Raman signal can be used to determine the extent of damage induced to the graphene and we focus here on the protective effect of the oxide coating.

Figure 3(a) shows representative Raman spectra for graphene after transfer, annealing and after $\mathrm{O}_{2}$ plasma exposure of graphene covered with $2.5,5,10,25 \mathrm{~nm}$ of $\mathrm{WO}_{3}$ and $\mathrm{MoO}_{3}$ (see supplementary material) ${ }^{33}$ respectively, and Figure 3(b) shows the corresponding relative changes in the $\mathrm{I}_{\mathrm{D}} / \mathrm{I}_{\mathrm{G}}$ ratio after $60 \mathrm{~s} \mathrm{O}_{2}$ plasma treatment as a function of the thermally evaporated metal oxide thickness. The Raman spectrum for graphene after transfer shows a characteristic $2 \mathrm{D}\left(\sim 2700 \mathrm{~cm}^{-1}\right)$, $\mathrm{G}\left(\sim 1600 \mathrm{~cm}^{-1}\right)$ and $\mathrm{D}\left(\sim 1350 \mathrm{~cm}^{-1}\right)$ peak and an $\mathrm{I}_{\mathrm{D}} / \mathrm{I}_{\mathrm{G}}$ ratio $\sim 0.05-10$ confirming the high quality of the starting material. ${ }^{12,13}$ Annealing in $\mathrm{H}_{2}$ is typically performed to remove the polymer residue post transfer which could otherwise short the OLED stack. However, the annealing typically introduces defects and can be seen in the form of an increase in the D peak with an $\mathrm{I}_{\mathrm{D}} / \mathrm{I}_{\mathrm{G}}$ ratio $\sim 0.15-0.18 .{ }^{18,19}$ For oxide coatings less than $10 \mathrm{~nm}$ in average thickness there is a drastic deterioration in the quality of graphene as highlighted by the large relative change in the $\mathrm{I}_{\mathrm{D}} / \mathrm{I}_{\mathrm{G}}$ ratio, i.e. $\mathrm{I}_{\mathrm{D}} / \mathrm{I}_{\mathrm{G}} \sim 1.34$ for $2.5 \mathrm{~nm}$ and $\mathrm{I}_{\mathrm{D}} / \mathrm{I}_{\mathrm{G}} \sim 0.30$ for $5 \mathrm{~nm}$ thick films of $\mathrm{WO}_{3}$, respectively. For $\mathrm{WO}_{3}$ thicknesses above $10 \mathrm{~nm}$ the relative change in the $\mathrm{I}_{\mathrm{D}} / \mathrm{I}_{\mathrm{G}}$ ratio is $\sim 0.06-0.02$, i.e. very small, and attributed to $\mathrm{O}_{2}$ ion diffusion through the side and pin-holes in the oxide layer. We note here that although $5 \mathrm{~nm}$ thick films of $\mathrm{WO}_{3}$ on graphene are continuous (see Figure 3(c), and TEM images shown elsewhere), ${ }^{19}$ they are unable to offer an effective protection from the $\mathrm{O}_{2}$ plasma at the given conditions to the graphene underneath. However, an increased oxide film thickness offers sufficient resistance against mass transfer for the radicals from the $\mathrm{O}_{2}$ plasma and prevents damage to the graphene. We observe a similar behavior for thermally evaporated $\mathrm{MoO}_{3}$ coatings, albeit with minor variation that probably originates from the differences in intrinsic material properties for the two different oxides. Even though $\mathrm{MoO}_{3}$ has attracted significant attention as a graphene and carbon nanotube dopant our choice of $\mathrm{WO}_{3}$ is based on its better stability to air exposure. ${ }^{9,18,19,31}$ Though, we emphasize that our method is highly relevant for a number of other optoelectronic applications using $\mathrm{MoO}_{3}$ as a p-type dopant for graphene and CNTs. In addition, $\mathrm{MoO}_{3}$ and $\mathrm{WO}_{3}$ have been identified as ITO sputtering protection layer in OLEDs and organic solar cells where a 40-60nm thick metal oxide layer can effectively prevent the sensitive organic layers from the high kinetic particle bombardment. ${ }^{34-36}$

We note that thermally evaporated metal oxides such as $\mathrm{MoO}_{3}$ and $\mathrm{WO}_{3}$ grow as sub-stoichiometric thin film which makes these materials n-type conductive due to oxygen vacancies. On the other hand, a very strong chemical reduction, e.g. as a result of high evaporation temperatures or adsorbates on the surface, leads to a new metal oxide composition with a high amount of $\mathrm{MoO}_{2}$ and $\mathrm{WO}_{2}$ sub-oxides. ${ }^{18,19,31,34}$ These species exhibit a low-band gap $(<2.5 \mathrm{eV})$ leading to an increased absorption of the metal oxide films. ${ }^{18,19,31,34} \mathrm{An}_{2}$ plasma might partially counteract this deficiency of metal oxides. X-ray 
photoelectron spectroscopy (XPS, $\mathrm{Mg}(\mathrm{Ka})$ radiation $^{18}$ source from a $\mathrm{Mg} / \mathrm{Al}$ double anode) shows that our $\mathrm{WO}_{3}$ layer is mainly composed of $\mathrm{W}^{6+}$ states and sub-stoichiometric $\mathrm{W}^{5+}$ states are at the detection limited (see supplementary material) ${ }^{37}$. The metal oxide composition is not affected by the plasma process. In addition, the XPS data confirms the p-doping of graphene upon $\mathrm{WO}_{3}$ deposition as seen in the corresponding graphene $\mathrm{C} 1 \mathrm{~s}$ core level shift of around $0.2 \mathrm{eV}$ towards lower binding energies. In a previous study on $\mathrm{MoO}_{3}$ doped graphene a similar shift of $0.25 \mathrm{eV}$ has been observed. ${ }^{18}$ For comparison, the Fermi level shift induced by metal oxide doping $\left(\mathrm{WO}_{3}\right.$ and $\left.\mathrm{MoO}_{3}\right)$ is significantly larger as reported for conventional $\mathrm{HNO}_{3}$ doping of graphene $(\sim 0.12 \mathrm{eV}) .{ }^{17}$ The XPS analysis also shows that the shift remains nearly unchanged before and after etching which again verifies that the doping of graphene is not affected by the $\mathrm{O}_{2}$ plasma process.

To demonstrate the efficiency of as-prepared graphene TCEs with reference to ITO electrodes, we fabricated OLED stacks as schematically outlined in Figure 4(a). We thereby compare two samples of graphene with a $5 \mathrm{~nm}$ and $15 \mathrm{~nm} \mathrm{WO}_{3}$ coating respectively. The thicknesses were chosen such that one sample is somewhat below and the other sample slightly above the critical oxide protection thickness for $\mathrm{O}_{2}$-plasma exposure, as identified above (see Figure 3(a) and (b)). Both graphene samples were $\mathrm{O}_{2}$-plasma patterned at $100 \mathrm{~W}$ for $2 \mathrm{~min}$ which is twice as long as required to fully etch unprotected graphene (see Figure 2(a)). In addition, the samples were shortly air-exposed before and after the plasma process. As reference electrode system, we use an ITO bottom electrode with a $15 \mathrm{~nm} \mathrm{WO}_{3}$ coating. For this ITO reference electrode no plasma treatment was applied and it was processed without breaking vacuum. All organic and inorganic layers were prepared by thermal evaporation in a custom-build deposition system at a base pressure of $10^{-6}$ mbar. The OLED stack comprises a $5 \mathrm{~nm}$ or $15 \mathrm{~nm} \mathrm{WO}_{3}$ layer, a $130 \mathrm{~nm} \mathrm{WO}_{3}$ doped 4,4'-Bis( $N$-carbazolyl)-1,1'-biphenyl (CBP) hole transport layer (20 wt\%), a $5 \mathrm{~nm}$ CBP hole transport layer, a $15 \mathrm{~nm}$ CBP doped with bis(2-phenylpyridine)(acetylacetonate)iridium(III) [Ir(ppy) 2 (acac)] (10 wt\%) emission layer, a 60nm 1,3,5-tris-phenyl-2-benzimidazolyl-benzene (TPBi) electron transport layer, a $1 \mathrm{~nm}$ 8-hydroxyquinolinato lithium (Liq) electron injection layer and a $100 \mathrm{~nm}$ Al top electrode.

Figure 4 summarizes the corresponding OLED device characteristics. The ITO-based control device shows the expected high efficiency with a steep rise of luminance with increasing voltage starting at $2.7 \mathrm{~V}$ and reaching $1000 \mathrm{~cd} / \mathrm{m}^{2}$ at around $4 \mathrm{~V}$. At this point a high power efficiency of $601 \mathrm{~m} / \mathrm{W}$ (without out-coupling structure) is obtained. In comparison, the graphene sample with $5 \mathrm{~nm} \mathrm{WO}_{3}$ coating shows a significantly higher operating voltage and therefore a reduced power efficiency of around $501 \mathrm{~m} / \mathrm{W}$ at $1000 \mathrm{~cd} / \mathrm{m}^{2}$. The voltage increase can be clearly attributed to a higher sheet resistance of the graphene caused by plasma damage, as we found $5 \mathrm{~nm} \mathrm{WO}_{3}$ not sufficient to provide an appropriate plasma protection (see Figure 3(a)). On the other hand, for the graphene electrode with $15 \mathrm{~nm} \mathrm{WO}_{3}$ the OLED device performance is nearly the same and up to $1000 \mathrm{~cd} / \mathrm{m}^{2}$ even slightly higher compared to the ITO-based control device. The somewhat higher operating voltage at very 
high luminance values $\left(>5000 \mathrm{~cd} / \mathrm{m}^{2}\right)$ of the $15 \mathrm{~nm} \mathrm{WO}_{3}$ graphene-based OLED is not attributed to damage, but to the limited sheet resistance of monolayer graphene compared to ITO, as we already reported elsewhere. ${ }^{18}$ To further reduce the sheet resistance 3-4 layers of highly doped graphene are needed. ${ }^{18}$ Nevertheless, we like to emphasize here that our method allows for the realization of efficient graphene-based OLEDs and despite air-exposure prior and after etching the device exhibits comparable performance to ITO-based devices.

\section{Conclusion}

Our data establishes a scalable integration route for graphene-based TCEs. We show that a thin oxide coating can fulfill a multifunctional role, providing effective graphene doping, ideal alignment of the transport levels at the graphene interface, effective wetting and graphene protection during oxygen plasma etching and patterning. We demonstrate OLED devices

based on patterned graphene-based TCEs without the use of lithography that have efficiencies comparable and possibly exceeding those of vacuum processed, ITO-electrode-based devices. Our approach is compatible with current industrial manufacturing processes and equally applicable to solution derived/exfoliated graphene and CNT electrodes as well as to a range of commonly used oxide films, such as $\mathrm{MoO}_{3}$. We think that such effective integration of nanomaterials is key to their emerging device technology.

\section{Acknowledgement}

Funding from EU FP7 programme Grafol (Grant No. 285275) and EPSRC (Grant No. EP/K016636/1, GRAPHTED) is acknowledged. P.R.K. acknowledges the Lindemann Trust Fellowship. 


\section{References}

(1) Gordon, R. G. C Riteria for Choosing Transparent Conductors. MRS Bull. 2000, 52-57.

(2) Novoselov, K. S.; Fal'ko, V. I.; Colombo, L.; Gellert, P. R.; Schwab, M. G.; Kim, K. A Roadmap for Graphene. Nature 2012, 490, 192-200.

(3) Hwang, J.; Kyw Choi, H.; Moon, J.; Yong Kim, T.; Shin, J.-W.; Woong Joo, C.; Han, J.-H.; Cho, D.-H.; Woo Huh, J.; Choi, S.-Y.; et al. Multilayered Graphene Anode for Blue Phosphorescent Organic Light Emitting Diodes. Appl. Phys. Lett. 2012, 100, 133304.

(4) Li, N.; Oida, S.; Tulevski, G. S.; Han, S.-J.; Hannon, J. B.; Sadana, D. K.; Chen, T.-C. Efficient and Bright Organic Light-Emitting Diodes on Single-Layer Graphene Electrodes. Nat. Commun. 2013, 4, 2294.

(5) Wang, Y.; Chen, X.; Zhong, Y.; Zhu, F.; Loh, K. P. Large Area, Continuous, Few-Layered Graphene as Anodes in Organic Photovoltaic Devices. Appl. Phys. Lett. 2009, 95, 063302.

(6) Wu, J.; Agrawal, M.; Becerril, H. A.; Bao, Z.; Liu, Z.; Chen, Y.; Peumans, P. Organic Light-Emitting Diodes on Solution-Processed Graphene Transparent Electrodes. ACS Nano 2010, 4, 43-48.

(7) Dean, C. R.; Young, A. F.; Meric, I.; Lee, C.; Wang, L.; Sorgenfrei, S.; Watanabe, K.; Taniguchi, T.; Kim, P.; Shepard, K. L.; et al. Boron Nitride Substrates for High-Quality Graphene Electronics. Nat. Nanotechnol. 2010, 5 , 722-726.

(8) Sun, T.; Wang, Z. L.; Shi, Z. J.; Ran, G. Z.; Xu, W. J.; Wang, Z. Y.; Li, Y. Z.; Dai, L.; Qin, G. G. Multilayered Graphene Used as Anode of Organic Light Emitting Devices. Appl. Phys. Lett. 2010, 96, 133301.

(9) Park, H.; Brown, P.; Bulović, V.; Kong, J. Graphene as Transparent Conducting Electrodes in Organic Photovoltaics: Studies in Graphene Morphology, Hole Transporting Layers, and Counter Electrodes. Nano Lett. 2011.

(10) Han, T.-H.; Lee, Y.; Choi, M.-R.; Woo, S.-H.; Bae, S.-H.; Hong, B. H.; Ahn, J.-H.; Lee, T.-W. Extremely Efficient Flexible Organic Light-Emitting Diodes with Modified Graphene Anode. Nat. Photonics 2012, 6, $105-110$.

(11) Wang, Y.; Tong, S. W.; Xu, X. F.; Ozyilmaz, B.; Loh, K. P. Interface Engineering of Layer-by-Layer Stacked Graphene Anodes for High-Performance Organic Solar Cells. Adv. Mater. 2011, 23, 1514-1518.

(12) Kidambi, P. R.; Ducati, C.; Dlubak, B.; Gardiner, D.; Weatherup, R. S.; Martin, M.; Seneor, P.; Coles, H.; Hofmann, S. The Parameter Space of Graphene Chemical Vapor Deposition on Polycrystalline Cu. J. Phys. Chem. C 2012, 116, 22492-22501.

(13) Kidambi, P. R.; Bayer, B. C.; Blume, R.; Wang, Z.-J.; Baehtz, C.; Weatherup, R. S.; Willinger, M.-G.; Schloegl, R.; Hofmann, S. Observing Graphene Grow: Catalyst-Graphene Interactions during Scalable Graphene Growth on Polycrystalline Copper. Nano Lett. 2013, 13, 4769-4778.

(14) Kidambi, P. R.; Bayer, B. C.; Weatherup, R. S.; Ochs, R.; Ducati, C.; Szabó, D. V.; Hofmann, S. Hafnia Nanoparticles - a Model System for Graphene Growth on a Dielectric. Phys. status solidi - Rapid Res. Lett. 2011, 5 , 341-343.

(15) Blume, R.; Kidambi, P. R.; Bayer, B. C.; Weatherup, R. S.; Wang, Z.-J.; Weinberg, G.; Willinger, M.-G.; Greiner, M.; Hofmann, S.; Knop-Gericke, A.; et al. The Influence of Intercalated Oxygen on the Properties of Graphene on Polycrystalline $\mathrm{Cu}$ under Various Environmental Conditions. Phys. Chem. Chem. Phys. 2014, 16, $25989-26003$. 
(16) Kobayashi, T.; Bando, M.; Kimura, N.; Shimizu, K.; Kadono, K.; Umezu, N.; Miyahara, K.; Hayazaki, S.; Nagai, S.; Mizuguchi, Y.; et al. Production of a 100-M-Long High-Quality Graphene Transparent Conductive Film by Roll-toRoll Chemical Vapor Deposition and Transfer Process. Appl. Phys. Lett. 2013, 102, 023112.

(17) Bae, S.; Kim, H.; Lee, Y.; Xu, X.; Park, J.; Zheng, Y.; Balakrishnan, J.; Lei, T.; Kim, H. R.; Song, Y. Il; et al. Rollto-Roll Production of 30-Inch Graphene Films for Transparent Electrodes. Nat. Nanotechnol. 2010, 5, 574-578.

(18) Meyer, J.; Kidambi, P. R.; Bayer, B. C.; Weijtens, C.; Kuhn, A.; Centeno, A.; Pesquera, A.; Zurutuza, A.; Robertson, J.; Hofmann, S. Metal Oxide Induced Charge Transfer Doping and Band Alignment of Graphene Electrodes for Efficient Organic Light Emitting Diodes. Sci. Rep. 2014, 4, 5380.

(19) Kuruvila, A.; Kidambi, P. R.; Kling, J.; Wagner, J. B.; Robertson, J.; Hofmann, S.; Meyer, J. Organic Light Emitting Diodes with Environmentally and Thermally Stable Doped Graphene Electrodes. J. Mater. Chem. C 2014, 2, 6940.

(20) Dlubak, B.; Kidambi, P. R.; Weatherup, R. S.; Hofmann, S.; Robertson, J. Substrate-Assisted Nucleation of UltraThin Dielectric Layers on Graphene by Atomic Layer Deposition. Appl. Phys. Lett. 2012, 100, 173113.

(21) Bult, J. B.; Crisp, R.; Perkins, C. L.; Blackburn, J. L. Role of Dopants in Long-Range Charge Carrier Transport for PType and N-Type Graphene Transparent Conducting Thin Films. ACS Nano 2013, 7, 7251-7261.

(22) Wang, D.-Y.; Huang, I.; Ho, P.; Li, S.; Yeh, Y.; Wang, D.; Chen, W.-L.; Lee, Y.; Chang, Y.; Chen, C.-C.; et al. Clean-Lifting Transfer of Large-Area Residual-Free Graphene Films. Adv. Mater. 2013, 25, 4521-4526.

(23) Degl'Innocenti, R.; Jessop, D. S.; Shah, Y. D.; Sibik, J.; Zeitler, J. A.; Kidambi, P. R.; Hofmann, S.; Beere, H. E.; Ritchie, D. A. Low-Bias Terahertz Amplitude Modulator Based on Split-Ring Resonators and Graphene. ACS Nano 2014, 8, 2548-2554.

(24) Butt, H.; Kidambi, P. R.; Dlubak, B.; Montelongo, Y.; Palani, A.; Amaratunga, G. A. J.; Hofmann, S.; Wilkinson, T. D. Visible Diffraction from Graphene and Its Application in Holograms. Adv. Opt. Mater. 2013, 1, 869-874.

(25) Badhwar, S.; Sibik, J.; Kidambi, P. R.; Beere, H. E.; Axel Zeitler, J.; Hofmann, S.; Ritchie, D. A. Intrinsic Terahertz Plasmon Signatures in Chemical Vapour Deposited Graphene. Appl. Phys. Lett. 2013, 103, 121110.

(26) Degl'Innocenti, R.; Jessop, D. S.; Shah, Y. D.; Sibik, J.; Zeitler, J. A.; Kidambi, P. R.; Hofmann, S.; Beere, H. E.; Ritchie, D. A. Terahertz Optical Modulator Based on Metamaterial Split-Ring Resonators and Graphene. Opt. Eng. 2014, 53, 057108.

(27) Baltazar, J.; Sojoudi, H.; Paniagua, S. A.; Zhang, S.; Lawson, R. A.; Marder, S. R.; Graham, S.; Tolbert, L. M.; Henderson, C. L. Photochemical Doping and Tuning of the Work Function and Dirac Point in Graphene Using Photoacid and Photobase Generators. Adv. Funct. Mater. 2014, 24, 5147-5156.

(28) Kim, K. K.; Reina, A.; Shi, Y.; Park, H.; Li, L.-J.; Lee, Y. H.; Kong, J. Enhancing the Conductivity of Transparent Graphene Films via Doping. Nanotechnology 2010, 21, 285205.

(29) Wu, Q.; Zhao, Y.; Hong, G.; Ren, J.; Wang, C.; Lee, S. T. Electronic Structure of MoO 3-X / Graphene Interface. 2013.

(30) Khrapach, I.; Withers, F.; Bointon, T. H.; Polyushkin, D. K.; Barnes, W. L.; Russo, S.; Craciun, M. F. Novel Highly Conductive and Transparent Graphene-Based Conductors. Adv. Mater. 2012, 24, 2844-2849.

(31) Meyer, J.; Hamwi, S.; Kröger, M.; Kowalsky, W.; Riedl, T.; Kahn, A. Transition Metal Oxides for Organic Electronics: Energetics, Device Physics and Applications. Adv. Mater. 2012, 24, 5408-5427. 
(32) Kidambi, P. R.; Blume, R.; Kling, J.; Wagner, J. B.; Baehtz, C.; Weatherup, R. S.; Schloegl, R.; Bayer, B. C.;

Hofmann, S. In Situ Observations during Chemical Vapor Deposition of Hexagonal Boron Nitride on Polycrystalline Copper. Chem. Mater. 2014, 141110130530003.

(33) See supplementary material at [URL will be inserted by AIP] for [Raman spectra showing degradation in quality of graphene underneath the $\mathrm{MoO}_{3}$ post-treated at $100 \mathrm{~W}$ for $60 \mathrm{~s}_{2}$ plasma treatment ].

(34) Meyer, J.; Winkler, T.; Hamwi, S.; Schmale, S.; Johannes, H.-H.; Weimann, T.; Hinze, P.; Kowalsky, W.; Riedl, T. Transparent Inverted Organic Light-Emitting Diodes with a Tungsten Oxide Buffer Layer. Adv. Mater. 2008, 20, 3839-3843.

(35) Schmidt, H.; Flügge, H.; Winkler, T.; Bülow, T.; Riedl, T.; Kowalsky, W. Efficient Semitransparent Inverted Organic Solar Cells with Indium Tin Oxide Top Electrode. Appl. Phys. Lett. 2009, 94, 243302.

(36) Meyer, J.; Görrn, P.; Hamwi, S.; Johannes, H.-H.; Riedl, T.; Kowalsky, W. Indium-Free Transparent Organic Light Emitting Diodes with Al Doped ZnO Electrodes Grown by Atomic Layer and Pulsed Laser Deposition. Appl. Phys. Lett. 2008, 93, 073308.

(37) See supplementary material at [URL will be inserted by AIP] for [XPS C1s and W4f data of graphene with a $15 \mathrm{~nm}$ $\mathrm{WO}_{3}$ protective layer which was $\mathrm{O}_{2}$-plasma treated at $100 \mathrm{~W}$ for $\left.2 \mathrm{~min}\right]$. 


\section{Figures}

a

(1)

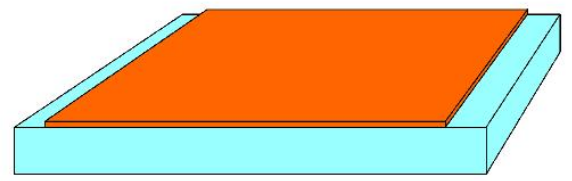

graphene transferred

(2)

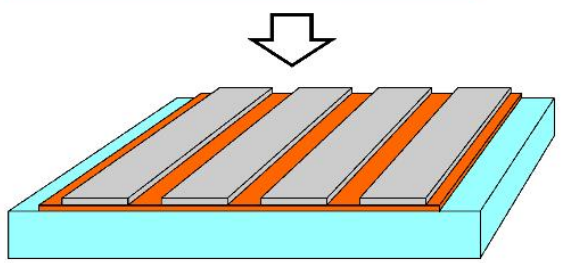

(3)

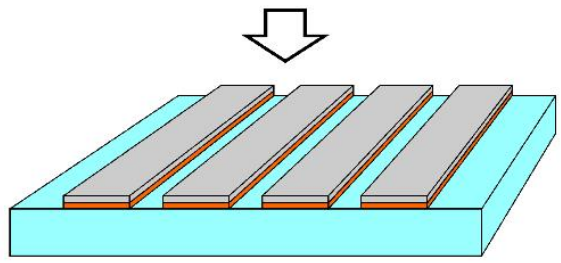

$\mathrm{O}_{2}$-plasma etching

(4)

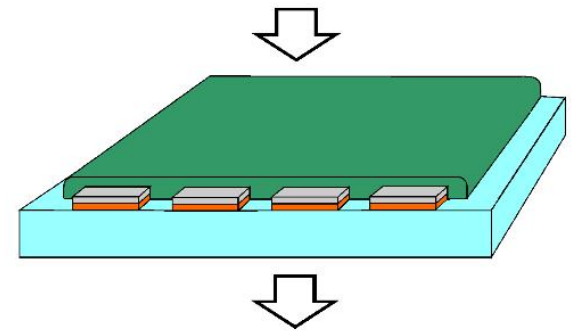

(5)

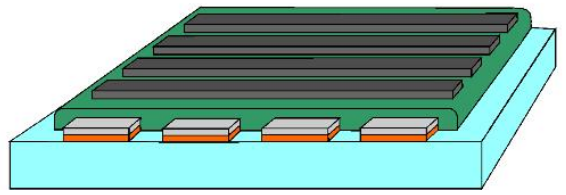
onto glass

$\mathrm{WO}_{3}$ deposition via shadow mask

b

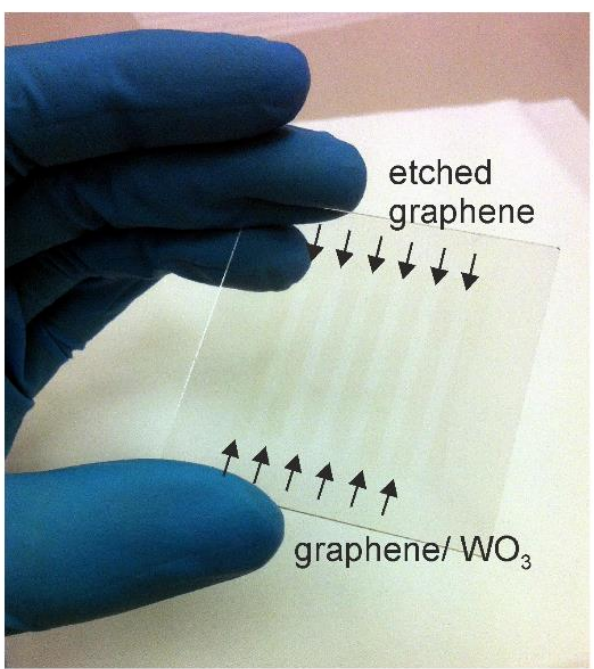

OLED stack deposition

\section{Metal deposition} via shadow mask

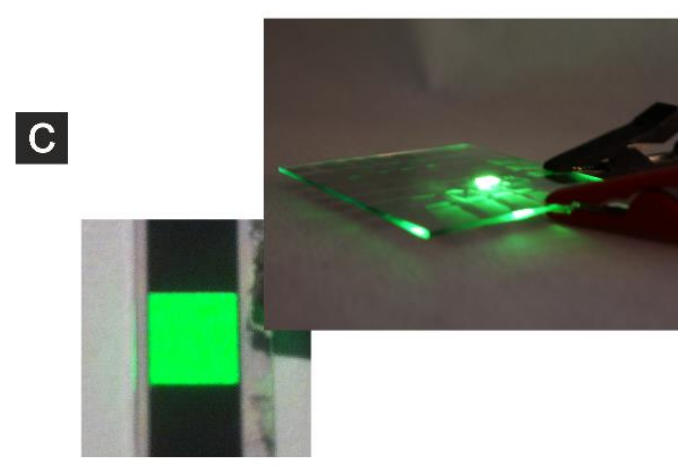

FIG 1. (a) The schematic fabrication flow diagram for OLEDs with metal oxide doped and subsequently patterned graphene electrode. (b) Photograph of $\mathrm{WO}_{3}$ patterned graphene as illustrated in step 3 of Figure 1(a). (c) Photograph of a $\mathrm{WO}_{3}$-plasma patterned OLED. Inset: Magnified view of the illuminating OLED area. 

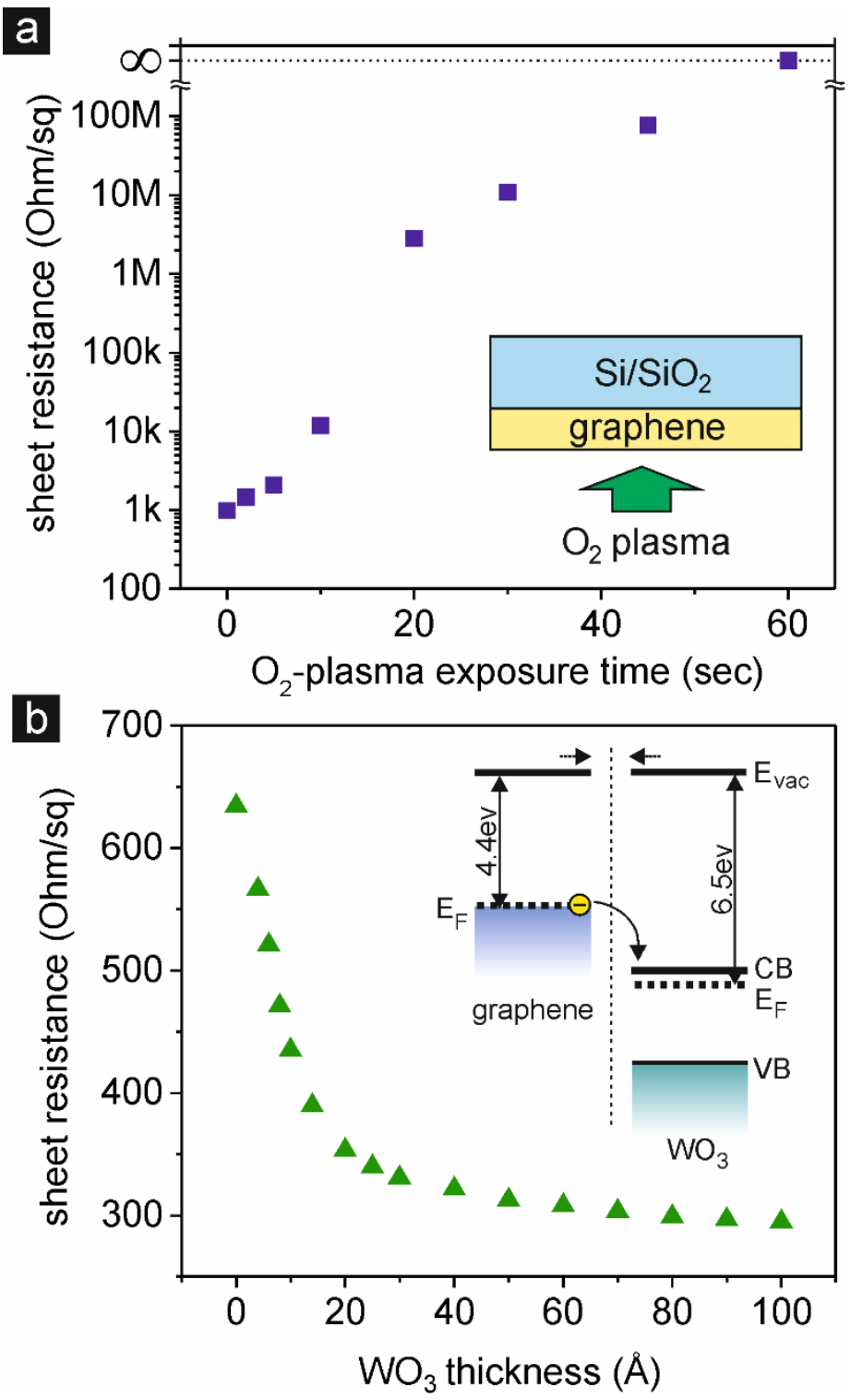

FIG 2. The change in sheet resistance of monolayer graphene transferred to a $\mathrm{SiO}_{2}(300 \mathrm{~nm}) / \mathrm{Si}$ wafer. (a) As a function of the $\mathrm{O}_{2}$ plasma (100W) exposure time (b) As a function of the $\mathrm{WO}_{3}$ layer thickness. Inset: Schematic illustration of charge transfer doping due to Fermi level alignment of graphene and $\mathrm{WO}_{3}$. 


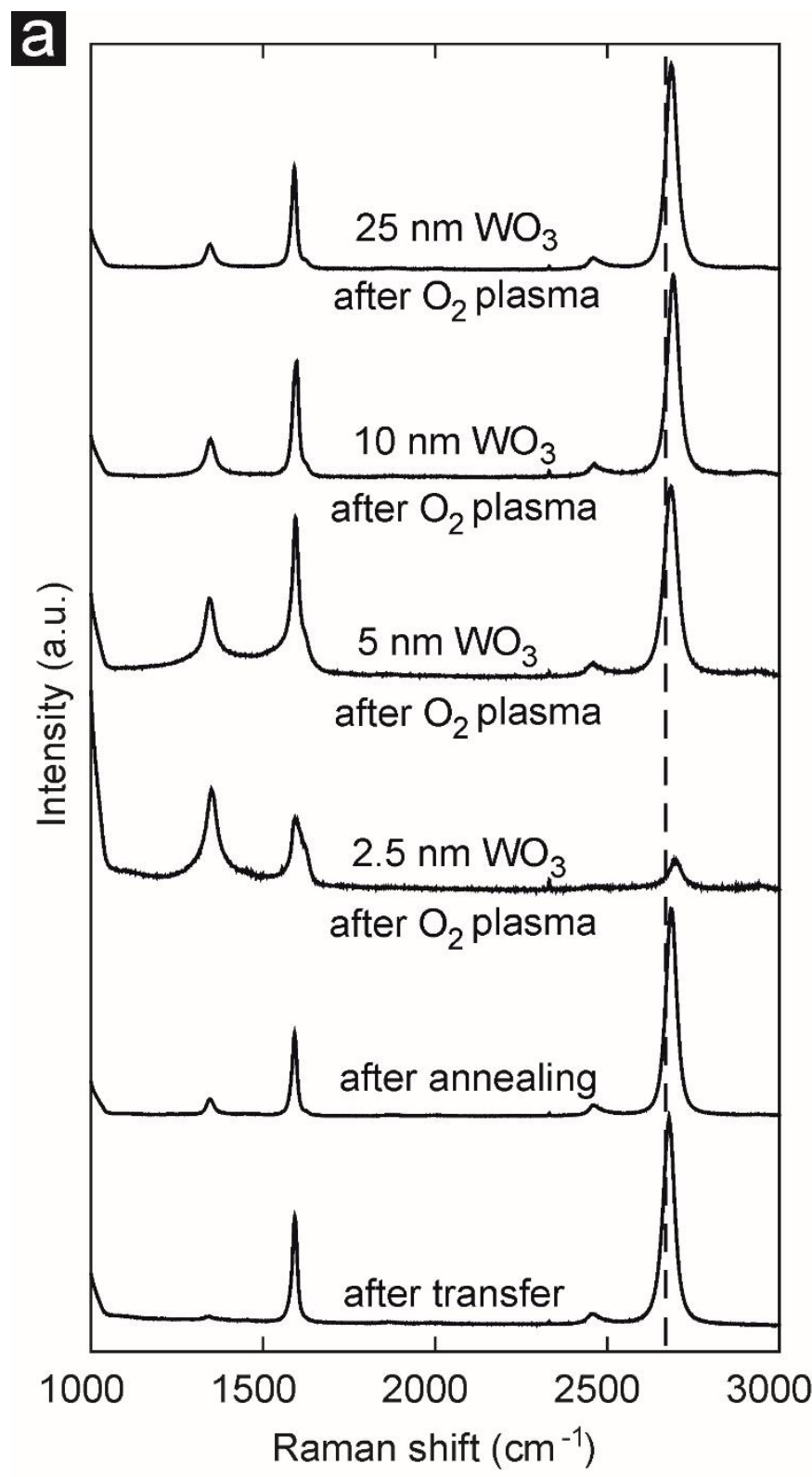

b
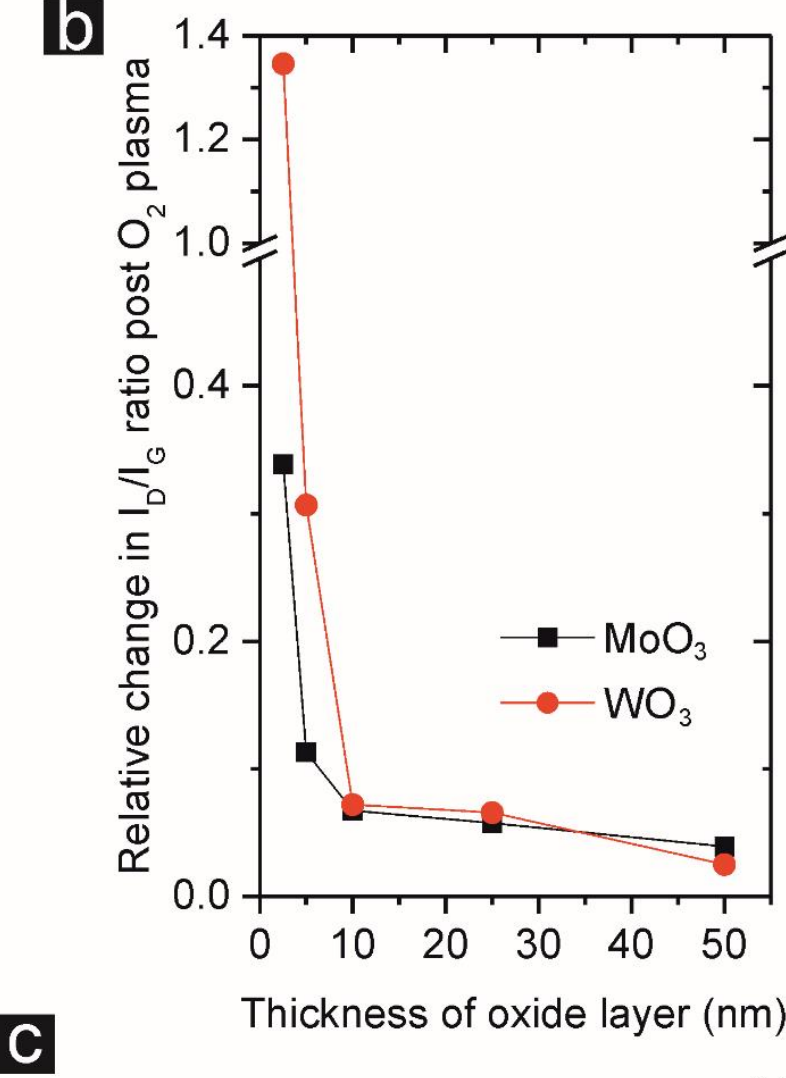

Thickness of oxide layer $(\mathrm{nm})$

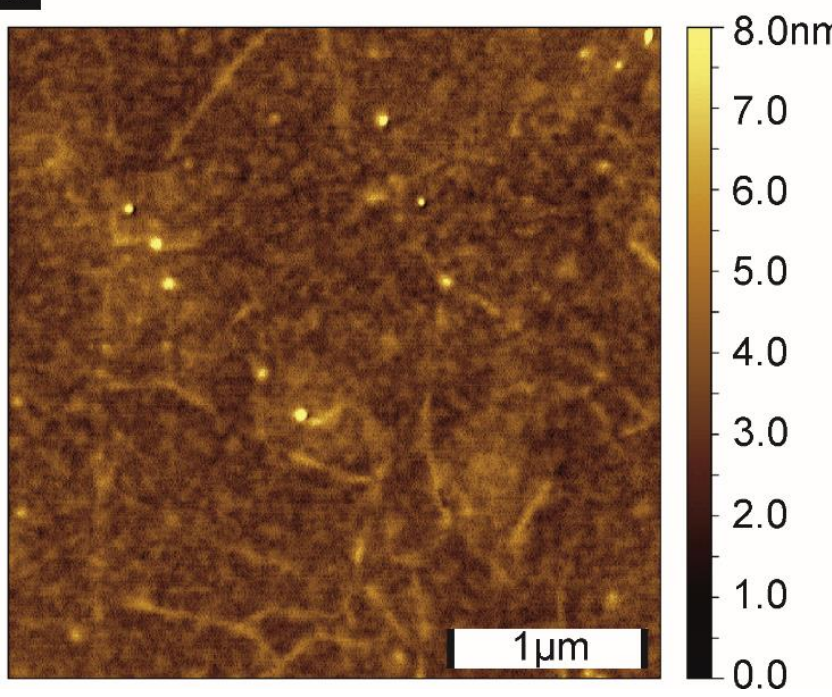

$8.0 \mathrm{~nm}$

5.0

4.0

3.0

.0

0

0.0

FIG 3. Thickness dependent protective nature of continuous $\mathrm{WO}_{3}$ and $\mathrm{MoO}_{3}$ films, respectively. (a) Representative Raman spectra of monolayer graphene underneath a $\mathrm{WO}_{3}$ layer post-treated at $100 \mathrm{~W}$ for $60 \mathrm{~s} \mathrm{O}_{2}$ plasma treatment (for $\mathrm{MoO}_{3}$ see supporting information). (b) Degradation in quality of graphene underneath the metal oxide as seen by the relative change in the Raman $\mathrm{I}_{\mathrm{D}} / \mathrm{I}_{\mathrm{G}}$ ratio as a function of metal oxide film thicknesses. (c) AFM image for a $5 \mathrm{~nm}$ thick layer of $\mathrm{WO}_{3}$ on graphene $\mathrm{SiO}_{2}(300 \mathrm{~nm}) / \mathrm{Si}$ wafer. 

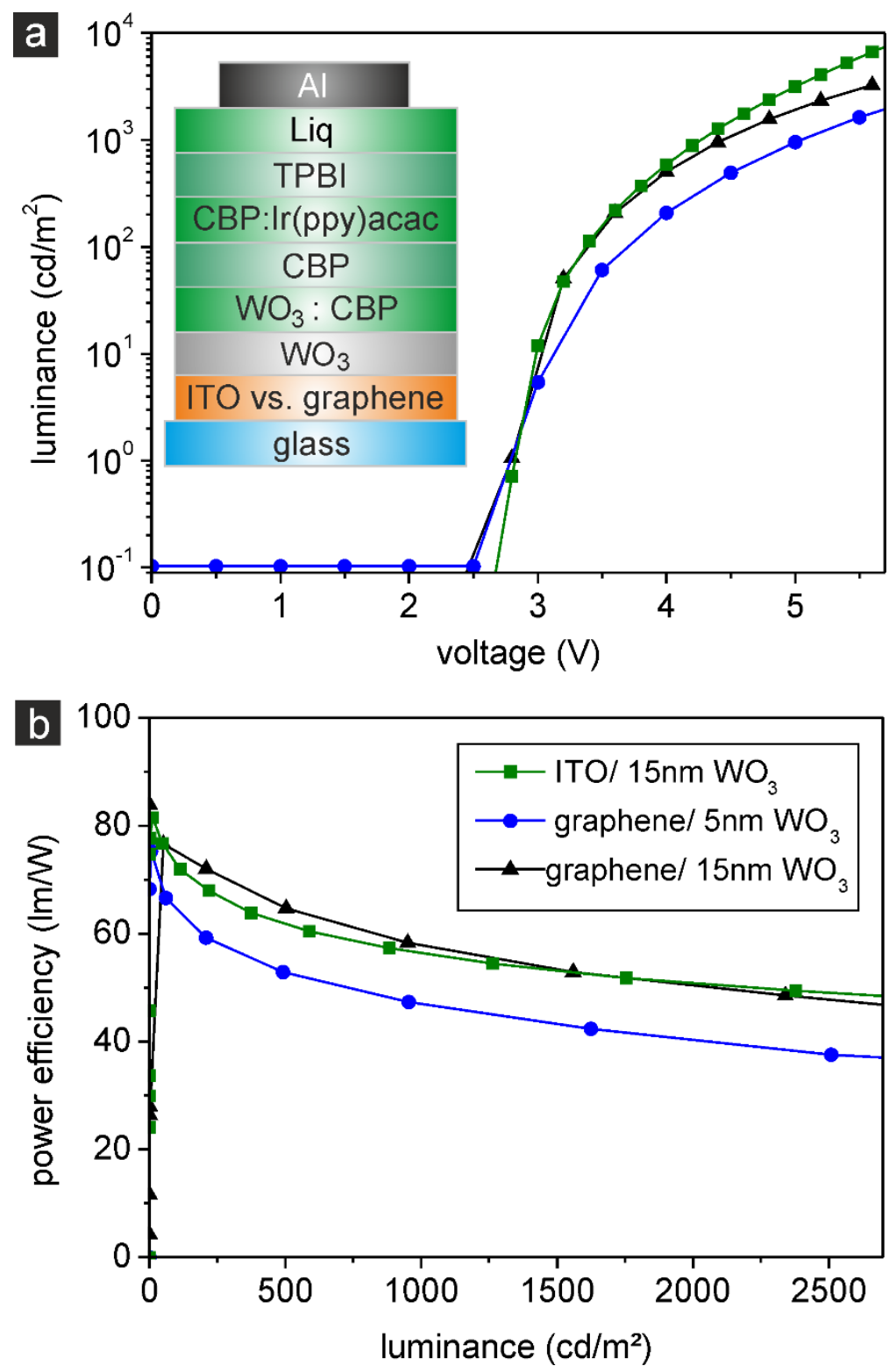

FIG 4. (a) Luminance as a function of the voltage. Inset: Schematic of the OLED stack. The $\mathrm{WO}_{3}$ layer thickness is $5 \mathrm{~nm}$ and $15 \mathrm{~nm}$, respectively. (b) Power efficiency as a function of the luminance for OLEDs with monolayer graphene electrodes doped with $\mathrm{WO}_{3}$. 